\title{
Composição florística de Plantas daninhas em um lago do Rio Solimões, Amazonas ${ }^{1}$
}

\author{
Floristic Composition of Weeds in a Lake of Solimoes River, Amazonas, Brazil
}

ALBERTINO, S.M.F. ${ }^{2}$, MILÉO, L.J. ${ }^{3}$, SILVA, J.F. ${ }^{4}$ e SILVA, C.A. ${ }^{5}$

\begin{abstract}
RESUMO - As áreas inundáveis localizadas na bacia dos rios Amazonas e Solimões são denominadas várzeas. A inundação é um evento natural que promove mudanças na estrutura e composição florística dessas comunidades. O conhecimento da diversidade de espécies é de fundamental importância para o entendimento da dinâmica da regeneração natural de espécies nos ecossistemas amazônicos. Este trabalho teve como objetivo levantar a composição florística do solo do fundo do lago do Manaquiri-AM, em um período de seca excepcional, ocorrida em 2005, na Amazônia. Foram realizadas coletas de material botânico em duas áreas do lago, em novembro de 2005; para a amostragem, utilizou-se um quadrado de madeira de $0,36 \mathrm{~m}^{2}$, atirado aleatoriamente por 20 vezes em cada local de estudo. A vegetação emergente foi de 5.958 indivíduos, distribuídos em sete famílias e nove espécies. As famílias mais representativas em número de espécies foram Poaceae e Cyperaceae. Cyperus esculentus e Luziola spruceana foram as mais frequentes, e Mimosa pudica e Alternanthera sessilis, as de maior abundância. C. esculentus e M. pudica apresentaram maior número de indivíduos, de densidade e de valor de importância. As espécies de plantas encontradas neste estudo mantiveram sua capacidade de crescer e se desenvolver mesmo após longo período submersas.
\end{abstract}

Palavras-chave: Amazônia, seca, planta daninha.

ABSTRACT - The swamps located at the basins of the Amazonas and Solimones rivers are denominated "várzeas". In these areas, flooding is a natural event that changes the structure and composition of the local plants. Thus, knowing the species diversity in these Amazon region areas is extremely important to understand the dynamics of the natural regeneration of the Amazon ecosystem species. Accordingly, the goal of this study was to survey the soil floristic composition at the bottom of the Manaquiri Lake, Amazon, during an exceptional dry period in 2005. Plants were collected in two areas of the lake in November 2005. Flora sampling was carried out using a $0.36 \mathrm{~m}^{2}$ wood frame, randomly cast 20 times at each site studied. The emergent vegetation at both collection sites was of 5,958 individuals distributed in seven families and nine species. The most representative families in number of species were Poaceae and Cyperaceae. Cyperus esculentus and Luziola spruceana were the most frequent, while Mimosa pudica and Alternanthera sessilis presented the highest abundance. C. esculentus and M. pudica had highernumber of individuals, density and value of importance. The plant species found in this study maintained their growing and developing capacity even after being submerged for a long period.

Keywords: Amazonia, dry period, weed.

1 Recebido para publicação em 16.6.2008 e na forma revisada em 18.2.2009.

2 Eng-agr-Ar ${ }^{\mathrm{a}}$, Doutoranda do Programa de Pós-Graduação em Agronomia Tropical, Universidade Federal do Amazonas - UFAM, 69077-000 Manuas-AM. <sonialbertino@ufam.edu.br>; ${ }^{3}$ Engo-Agro $^{0}$, M.Sc., Professor Assistente - UFAM, Campus de Ciências Agrárias e Ambientais Benjamim Constant, <AM.libiamileo@ufam.edu.br >; ${ }^{4}$ Engo-Agro ${ }^{\circ}$, Dr., Prof. Associado - UFAM, Faculdade de Ciências Agrárias, Dep. de Produção Animal e Vegetal, Av. Gal. Rodrigo Octavio J.Ramos, 3000, 69077-000 Manaus-AM, <jfsivla@ufam.edu.br>; ${ }^{5}$ Bal. Ciên. Sociais - UFAM, Centro de Ciências do Ambiente, Av. Gal. Rodrigo Octavio J. Ramos, 3000, 69077-00 Manaus-AM.

Planta Daninha, Viçosa-MG, v. 27, n 1, p. 1-5, 2009 


\section{INTRODUÇÃO}

No Amazonas, duas estações climáticas ocorrem durante o ano: uma seca, no período de julho a outubro, e outra chuvosa, de novembro a junho. As áreas próximas às margens dos rios sofrem inundações periódicas anuais, determinando períodos alternados de cheia e vazante (Viers et al., 2005). As áreas inundáveis, nas bacias dos rios Amazonas e Solimões, são denominadas várzeas e ocupam uma área superior a 300.000 km² (Junk, 1985).

A variação no fluxo da inundação anual do sistema dos rios Amazonas/Solimões/ Negro, nas proximidades de Manaus, pode ser constatada nas médias anuais da série de 103 anos de observações do Serviço Geológico do Brasil; a maior cheia ocorreu no mês de junho de 1953 (29,69 m), e a maior vazante (seca), em outubro de 1963 (13,64 m) (CPRM, 2005).

O ano deste estudo (2005) foi excepcionalmente seco, embora antecedido de uma cheia considerada mediana $(28,10 \mathrm{~m})$ (CPRM, 2005). O menor nivel - observado em 2005 foi de 14,75 m, nos dias 25 e 26 de outubro; no dia 11 de novembro do mesmo ano, o nivel estava em $16,61 \mathrm{~m}$, indicando o início do período de recuperação do nível do rio (ANA, 2005).

A distribuição e a diversidade das espécies de plantas em ambientes inundáveis variam dentro e entre os diferentes hábitats, de acordo com os gradientes de inundação (Nunes da Cunha \& Junk, 2001).
A inundação é responsável por mudanças na estrutura e composição florística nesses locais (Schessl, 1999); portanto, estudo sobre a manutenção da diversidade de espécies e sua regeneração natural permite previsões sobre o comportamento e desenvolvimento da flora, pois fornece a relação e a quantidade de espécies que constituem seu estoque, bem como sua distribuição na área.

Estudos sobre composição florística em ecossistemas amazônicos se restringem basicamente a florestas de terra firme e áreas agrícolas. Em áreas de várzea, poucos estudos foram realizados, como os de Santos \& Jardim (2006), Gama et al. (2002) e Higuchi et al. (1985).

Este trabalho teve como objetivo estudar a composição florística de plantas daninhas no lago do Manaquiri, AM, no período da seca de 2005.

\section{MATERIAL E MÉTODOS}

Este estudo foi realizado em duas áreas do lago do Manaquiri, localizado à margem direita do rio Solimões, nas comunidades de São Francisco (S 03 26’38.3" W 60 31'21.2") e Terra Preta (S 03 25’21.6" W 60 29'49.6"), ambas no município de Manaquiri, Estado do Amazonas (Figura 1). Esse lago permanece inundado durante todo o ano, variando sua profundidade de acordo com a época de seca ou de chuva. Na época da cheia do rio Solimões, sua profundidade média é de $25 \mathrm{~m}$ e, na vazante, de 1,5 m. Às margens do lago existem

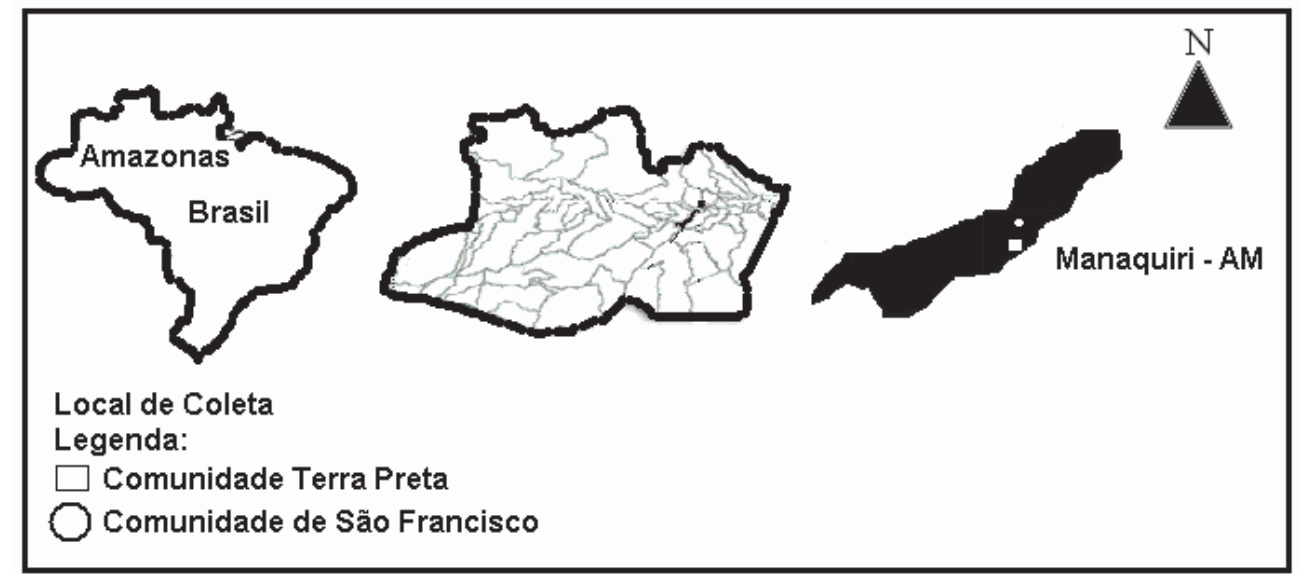

Figura 1 - Localização da área de estudo, no leito do lago do Manaquiri, Estado do Amazonas. 
áreas cultivadas com culturas alimentícias e pastagem de pequenos agricultores.

As coletas das plantas do lago foram em novembro de 2005, já no fim da estação seca. As espécies de plantas foram amostradas com um quadrado de madeira de $0,36 \mathrm{~m}^{2}$, atirado aleatoriamente por 20 vezes em cada comunidade, perfazendo um total de 40 amostras e uma área amostral de $14,4 \mathrm{~m}^{2}$.

As espécies coletadas foram identificadas por comparações com espécimes da coleção do Laboratório de Ciência das Plantas Daninhas da Universidade Federal do Amazonas (UFAM), com bibliografias especializadas e auxílio de especialistas.

Os parâmetros fitossociológicos medidos para as espécies foram: frequência $($ Fre $)=$ número de parcelas que contêm a espécie/ número total de parcelas utilizadas; densidade (Den) = número total de indivíduos por espécie/ área total coletada; abundância $(\mathrm{Abu})=$ número total de indivíduos por espécie/número total de parcelas que contêm a espécie; frequência relativa $($ Frer $)$ = frequência da espécie $\mathrm{x} 100 /$ frequência total de todas as espécies; densidade relativa $($ Der $)=$ densidade da espécie x 100/densidade total de todas as espécies; abundância relativa $(\mathrm{Abr})=$ abundância da espécie $\times 100 /$ abundância total de todas as espécies; e índice de valor de importância $(I V I)=$ Frer + Der + Abr (Brandão et al., 1998).

\section{RESULTADOS E DISCUSSÃO}

As espécies encontradas nos lagos do rio Solimões e seus parâmetros fitossociológicos foram listados por ordem de valor de importância e encontram-se na Tabela 1.

A vegetação emergente nos dois locais de coleta foi de 5.958 indivíduos, distribuídos em sete famílias, representadas por nove espécies: cinco da classe das dicotiledôneas e quatro das monocotiledôneas.

O baixo número de famílias e espécies nesse ecossistema pode estar condicionado ao próprio ambiente; por se tratar de um leito de lago, somente espécies adaptadas conseguem sobreviver e também manter suas sementes viáveis durante longos períodos submersas.

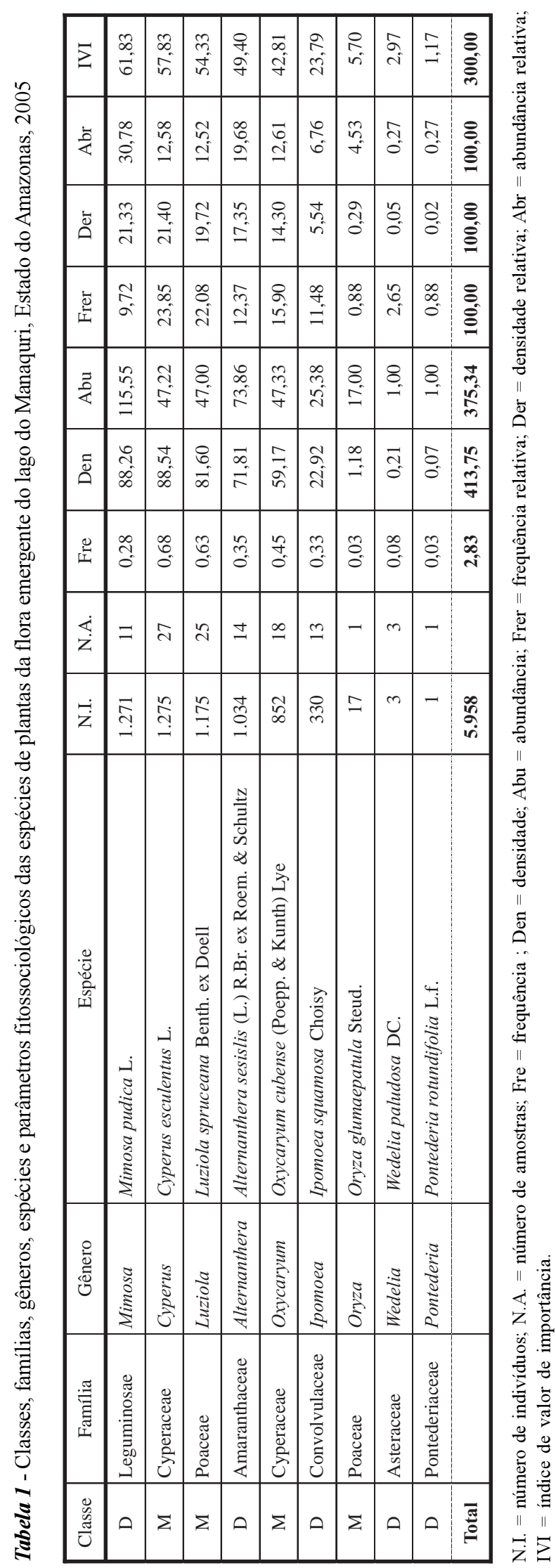

Planta Daninha, Viçosa-MG, v. 27, n 1, p. 1-5, 2009 
Nesse lago, a última seca ocorrida foi em 1963, quando o seu leito foi exposto à condição de ar e luz solar (CPRM, 2005).

Scarano et al. (1994) afirmaram que a sobrevivência vegetal nessas áreas não depende apenas de um único mecanismo adaptativo. Já Bornette et al. (1998) consideram que a redução do nível da água é responsável pela baixa diversidade de plantas, pois reduz a conectividade entre os ambientes, aumentando a competição e impedindo o aporte de propágulos que são dispersos, principalmente, pela água. O aparecimento das espécies do leito do lago do Manaquiri, provavelmente, tenha sido favorecido pela sua exposição às condições de ar e luz, como consequência do período de seca, na Amazônia, naquele ano.

As famílias mais representativas em número de espécies foram Poaceae e Cyperaceae, com duas espécies cada. A ocorrência dessas famílias com maior número de espécies é compativel com o encontrado por Nunes da Cunha et al. (2001) em levantamento de espécies de campo inundável, onde registraram dez espécies para Poaceae e cinco para Cyperaceae. Schessl (1999) constatou, em regiões alagáveis do Mato Grosso, que Poaceae, Leguminosae e Cyperaceae foram as famílias que concentraram maior número de espécies. Crispim et al. (2002) verificaram aumento na dispersão de Cyperaceae após inundação em campos do Pantanal (MS) e, nos meses de seca, maior participação das Poaceae.

Cyperus esculentus (1.275) e Mimosa pudica (1.271) foram as espécies que apresentaram maior número de indivíduos e também as de maior densidade, $\operatorname{com} 88,54$ e 88,26 plantas $\mathrm{m}^{-2}$, respectivamente. Como as mais abundantes, destacaram-se $M$. pudica $(115,55)$ e Alternanthera sessilis $(73,86)$.

Já quanto à frequência relativa a espécies, C. esculentus $(23,85 \%)$ seguida de Luziola spruceana $(22,08 \%)$ registraram maiores valores. Essas duas espécies, juntas, apresentaram, visualmente, dominância sobre as demais no lago, o que pode ser constatado pela ocorrência em 27 e 25 amostras das 40 analisadas, respectivamente. Em áreas agrícolas de várzea no Estado do Tocantins, C. esculentus também apresentou maior densidade relativa $(40,65 \%)$ e maior índice de importância relativa $(91,4)$ (Erasmo et al., 2004).

As espécies com maior índice de valor de importância foram M. pudica (Leguminosae) e C. esculentus (Cyperaceae), com IVI de 61,83 e 57,83 , respectivamente. Segundo Moreira \& Silva (1993), a presença de Leguminosae em solos inundáveis é vantajosa para esta família, pela associação que possui com a bactéria Rhizobium, como estratégia para compensar o déficit de nitrogênio nesses solos.

Essas duas espécies apresentaram grande ocorrência no ambiente do lago, com coloração verde e produção de sementes mesmo após a subida da água (Figura 2).

As espécies de plantas encontradas neste estudo mantiveram sua capacidade de crescer e se desenvolver mesmo após longo período submersas.

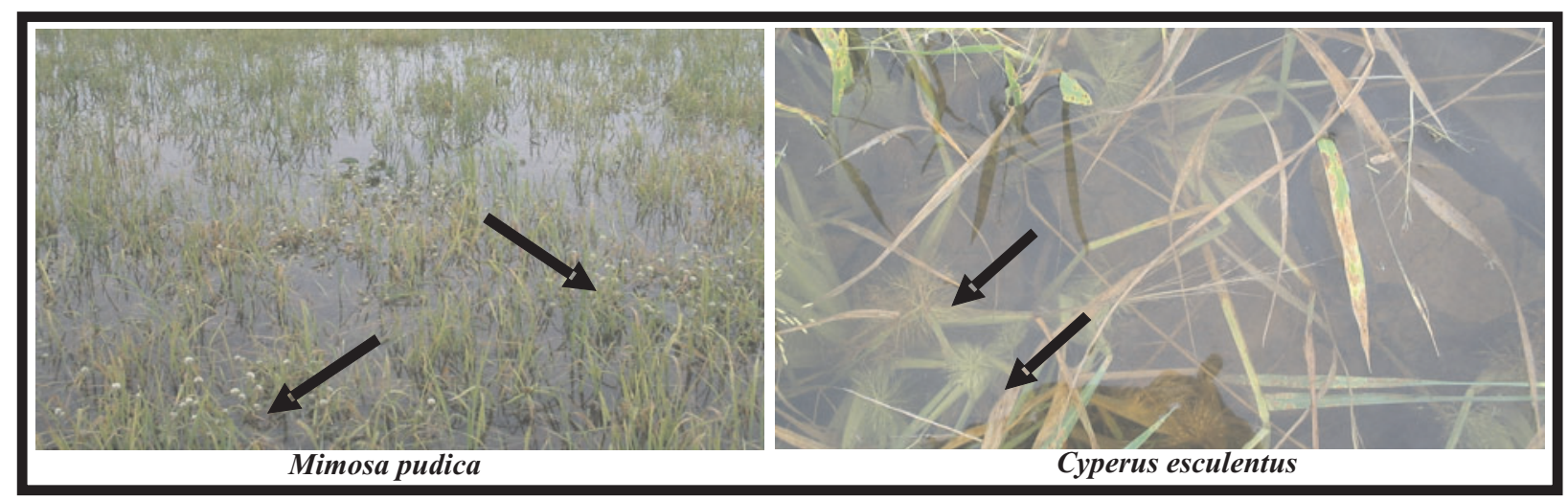

Figura 2 - Aspecto de Mimosa pudica e Cyperus esculentus no lago do Manaquiri, Estado do Amazonas, no início do período chuvoso. 


\section{LITERATURA CITADA}

AGÊNCIA NACIONAL DE ÁGUAS - ANA. Boletim de monitoramento da seca na região Amazônica, 2005.

Disponível em: <http//:www.ana.gov.br>. Acesso em: 31 de maio de 2008.

BORNETTE, G.; AMOROS, C.; LAMOUROUX, N. Aquatic plant diversity in riverine wetlands: the role of connectivity. Fresh. Biol., v. 39, n. 2, p. 267-283, 1998.

BRANDÃO, M., BRANDÃO, H.; LACA-BUENDIA, J. P. A mata ciliar do rio Sapucaí-MG: fitossociologia. Daphne, v. 8, n. 4, p. 36-48, 1998.

CRISPIM, S. M. A. et al. Variação sazonal na frequência e composição botânica em área de máxima inundação, PantanalMS, Brasil. Arch. Zootecnia, v. 51 p. 149-160, 2002.

COMPANHIA DE PESQUISA DE RECURSOS MINERAIS - CPRM. Serviço Geológico do Brasil. Relatório da seca de 2005. Disponível em: $<\mathrm{http} / /$ :www.cprm.gov.br $>$. Acesso em: 5 de junho de 2006.

ERASMO, E. A. L.; PINHEIRO, L. L.; COSTA, N. V. Levantamento fitossociológico das comunidades de plantas daninhas infestantes em áreas de produção de arroz irrigado cultivado sob diferentes sistemas de manejo. Planta Daninha, v. 22, n. 2. p. 195-201, 2004.

GAMA, J. R. V.; BOTELHO S. A.; BENTES-GAMA, M. M. Composição florística e estrutura da regeneração natural de floresta secundária de várzea baixa no estuário amazônico. R. Árvore, v. 26, n. 5, p. 559-566, 2002.

HIGUCHI, N. et al. Bacia 3-Inventário diagnóstico da regeneração natural. Acta Amaz., v. 15, n. 1-2, p. 199-233, 1985.
JUNK, W. J. The Amazon flood plain a sink or source for organic carbon? Mitteilungen aus dem. GeologischePalaontologischen Institut der Universitat Hamburg, v. 58 , p. $267-283,1985$

MOREIRA, F. M. S.; SILVA, M. F. Associação rizóbioleguminosas na Amazônia. B. Museu Paraense Emílio Goeldi, Série Bot., v. 9, n. 2, p. 129-141, 1993.

NUNES DA CUNHA, C.; JUNK, W. J. Distribution of wood plant communities along the flood gradient in the Pantanal of Poconé, Mato Grosso, Brazil. Inter. J. Ecol. Environ., v. 27, n. 1, p. 63-70, 2001

NUNES DA CUNHA, C.; VILHALVA, D. A. A.; FERREIRA, H. Espécies de campo inundável e de brejo, Fazenda Retiro Novo, Pantanal de Poconé, MT. In: SIMPÓSIO SOBRE RECURSOS NATURAIS E SÓCIOECONÔMICOS DO PANTANAL - OS DESAFIOS DO NOVO MILÊNIO, 3., Corumbá, 2000. Anais... Brasília, Embrapa-CPAP., 2001. p. 1-14.

SCHESSL, M. Floristic composition and structure of floodplain vegetation in northern Pantanal of Mato Grosso, Brasil. Phyton, v. 39, n. 2, p. 303-336. 1999.

SANTOS, G. C.; JARDIM, M. A. G. Florística e estrutura do estrato arbóreo de uma floresta de várzea no município de Santa Bárbara do Pará, Estado do Pará, Brasil. Acta Amaz., v. 36, n. 4 , p. $437-446,2006$.

SCARANO, F. R.; CATTÂNIO, J. H.; CRAWFORD, R. M. $\mathrm{M}$. Root carbohidrate storage in young samplings of an Amazonian tidal várzea forest before the onset of wet season. Acta Bot. Bras., v. 8, n. 2, p. 129-139, 1994.

VIERS, J. et al. The influence of the Amazonian floodplain ecosystems on the trace elementes dynamics of the Amazon River maistem (Brazil). Sci. Total Environ., v. 339, n. 2, p. 219-232, 2005. 